\title{
Polar Interactions Play an Important Role in the Energetics of the Main Phase Transition of Phosphatidylcholine Membranes
}

\author{
Alvaro Garcia, ${ }^{\dagger, \S}$ Haipei Zou, ${ }^{\dagger}$ Khondker R. Hossain, $^{\dagger, \dagger}$ Qikui Henry Xu, ${ }^{\dagger}$ Annabelle Buda, ${ }^{\dagger}$ \\ and Ronald J. Clarke*,t, \\ ${ }^{\dagger}$ School of Chemistry, University of Sydney, Sydney, New South Wales 2006, Australia \\ ${ }^{\ddagger}$ The University of Sydney Nano Institute, Sydney, New South Wales 2006, Australia
}

Supporting Information

\begin{abstract}
Conformational changes of membrane proteins are accompanied by deformation in the surrounding lipid bilayer. To gain insight into the energetics of membrane deformation, the phase behavior of dimyristoylphosphatidylcholine (DMPC) membranes in the presence of the dipole potential, $\psi_{\mathrm{d}}$, modifiers was investigated by differential scanning calorimetry. 7-Ketocholesterol, which weakens $\psi_{\mathrm{d}}$ and reduces membrane-perpendicular dipole-dipole repulsion, causes a discrete second peak on the high-temperature side of the main transition, whereas 6-ketocholestanol, which strengthens $\psi_{\mathrm{d}}$ and increases membrane-perpendicular dipole-dipole repulsion, merely produces a shoulder. Measurements on pure DMPC vesicles showed that the observed

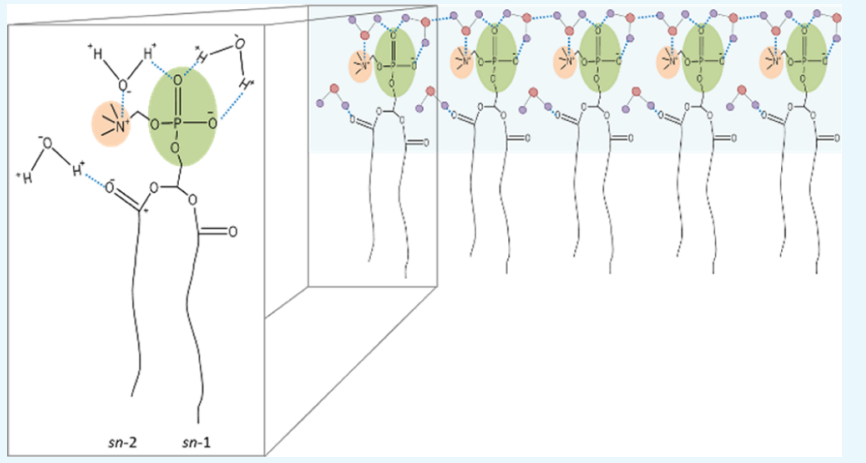
temperature profile could not be explained by a single endothermic process, that is, breaking of van der Waals forces between hydrocarbon chains alone. Removal of $\mathrm{NaCl}$ from the buffer caused an increase in the main transition temperature and the appearance of an obvious shoulder, implicating polar interactions. Consideration of the phosphatidylcholine (PC) head group dipole moment indicates direct interactions between PC dipoles that are unlikely to account for the additional process. It seems more likely that the breaking of an in-plane hydrogen-bonded network consisting of hydrating water dipoles together with zwitterionic lipid head groups is responsible. The evidence presented supports the idea that the breaking of van der Waals forces between lipid tails required for the main phase transition of PC membranes is coupled to partial breaking of a hydrogen-bonded network at the membrane surface.
\end{abstract}

\section{INTRODUCTION}

The phospholipid component of biological cell membranes contains multiple dipolar residues within the head group region of the membrane (e.g., ester carbonyl groups, water dipoles, and phosphocholine), which could via polar interactions play important roles in determining membrane stability and in modulating the behavior of membrane proteins. However, the contributions of polar interactions to membrane function are, up to now, not well understood.

Let us consider first the dipolar arrangement perpendicular to the surface of the membrane. The net effect of the perpendicular components of all dipolar groups produces an electrical potential difference, the membrane dipole potential, $\psi_{\mathrm{d}}$, which is located in the narrow region between the glycerol backbone of the phospholipids and the interface with the neighboring aqueous solution. ${ }^{1-4}$ The concept of the dipole potential is widely accepted, even though research on its implications for biological membrane function has been limited. From first principles, it is logical that the anisotropic arrangement of any lipid bilayer structure must necessarily require an alignment of the lipid head groups and that from this alignment of the associated dipolar groups, an electrical potential should arise. Because $\psi_{\mathrm{d}}$ drops over a small distance, it produces very large field strengths of $10^{8}$ to $10^{9} \mathrm{~V} \mathrm{~m}^{-1}$. This is far in excess of field strengths typically produced by the transmembrane electrical potential, that is, $\sim 10^{7} \mathrm{~V} \mathrm{~m} \mathrm{~m}^{-1}$. However, in spite of the large field strength $\psi_{\mathrm{d}}$ produces, it appears to have little effect on the binding and conduction of ions through ion channels or pumps. The reason for this is that, except for small pore-forming peptides such as gramicidin, the ions are largely shielded from the high electric field by the intervening protein mass. ${ }^{5,6}$

Nevertheless, significant effects of $\psi_{\mathrm{d}}$ on the kinetics of both ion pumps, such as $\mathrm{Na}^{+}, \mathrm{K}^{+}$-ATPase, ${ }^{7}$ and ion channels, such as the voltage-gated hERG potassium channel, ${ }^{8}$ have been reported. The effects of $\psi_{\mathrm{d}}$ on these proteins appear to occur via an effect on the kinetics of protein conformational changes, that is, gating in the case of ion channels. This is consistent with shielding of the transported ions from the electric field produced by $\psi_{\mathrm{d}}$, as described above. The effect of $\psi_{\mathrm{d}}$ on

Received: November 12, 2018

Accepted: December 26, 2018

Published: January 8, 2019 
membrane protein conformational changes has been postulated $^{9}$ to be due to local bending of the membrane surface around membrane proteins, needed to accommodate changes in protein hydrophobic thickness associated with the proteins' conformational changes. Any membrane bending must cause changes in lipid packing density which produces changes in $\psi_{\mathrm{d}}$ and in the energy of dipole-dipole repulsion perpendicular to the membrane surface.

Phospholipid membranes, however, do not only have dipoles arranged perpendicular to the membrane surface. ${ }^{31} \mathrm{P}$ NMR data and neutron diffraction studies have shown that the $\mathrm{P}-\mathrm{N}$ dipole of the phosphatidylcholine (PC) or phosphatidylethanolamine head group lies on an average approximately parallel to the membrane surface, ${ }^{10,11}$ but with small tilts of the dipole relative to the membrane surface caused by changes in lipid hydration or the binding of ions or dipolar compounds to the membrane surface. ${ }^{12-15}$ In addition, both X-ray crystallographic data ${ }^{16}$ and infrared spectroscopic data ${ }^{15,17-20}$ indicate that the phosphate group in particular, but also the nitrogen of the choline residue, are hydrogen bonded to water dipoles within the surface, leading to a cooperative hydrogen-bonded network. $^{16,20}$ Depending on the arrangement of neighboring head groups relative to one another, the $\mathrm{P}-\mathrm{N}$ dipoles and the water dipoles could interact with one another attractively or repulsively. Whichever arrangement the head groups and their associated water adopt, the overall effect of dipolar forces on membrane function must be because of the net result of both membrane-perpendicular and membrane-parallel components of all lipid-associated and hydrating water dipole moments.

To definitively attribute any change in behavior of a membrane protein to a membrane-mediated effect arising from the dipolar structure of the lipid membrane, and to exclude all other possibilities, is exceedingly difficult. Therefore, rather than study the effect of membrane dipoles on the deformation of a membrane caused by the conformational change of a protein, we have chosen to investigate a simpler model system, where it is known that a significant change in membrane hydrophobic thickness occurs, that is, the gel-toliquid-crystalline phase transition of PC membranes. As the membrane undergoes the transition from the gel to the liquidcrystalline phase, the breaking of van der Waals forces between the hydrocarbon chains converts them from an all-trans state to a much more fluid and disordered state with a continually changing mixture of trans and gauche conformations. The disordering of the hydrocarbon chains increases the crosssectional area per lipid and causes a thinning of the membrane. ${ }^{21-25}$ Thus, the phase transition results in a significant reduction in hydrophobic thickness. For dimyristoylphosphatidylcholine (DMPC), the decrease in total membrane thickness on passing from the gel to the liquid crystalline phase has been determined by X-ray diffraction to be approximately $21 \%$, that is, from $44.5 \AA$ in the gel phase to $35 \AA$ in the liquid-crystalline phase. ${ }^{21}$ If dipole-dipole interactions do indeed affect the energetics of membrane bending, then the gel-to-liquid-crystalline phase transition should be dependent on the presence of known dipole potential modifiers. Not only was this found here to be the case but also the results indicate that the weakening of in-plane polar attraction in the gel phase is an important contributor to the energetics of the transition. Thus, contrary to common belief, the transition is not purely due to the breaking of the van der Waals forces between the hydrocarbon tails.

\section{RESULTS}

Gel-to-Liquid-Crystalline Phase Transition of Mixed DMPC/Sterol Membranes. Cholesterol and its derivatives are known to have significant effects on the magnitude of $\psi_{\mathrm{d}}$ and have often been used as tools to modify $\psi_{\mathrm{d}}{ }^{26-28}$ Here, we used cholesterol itself and two derivatives, 6-ketocholestanol and 7-ketocholesterol (see Figure 1). Cholesterol and 6-
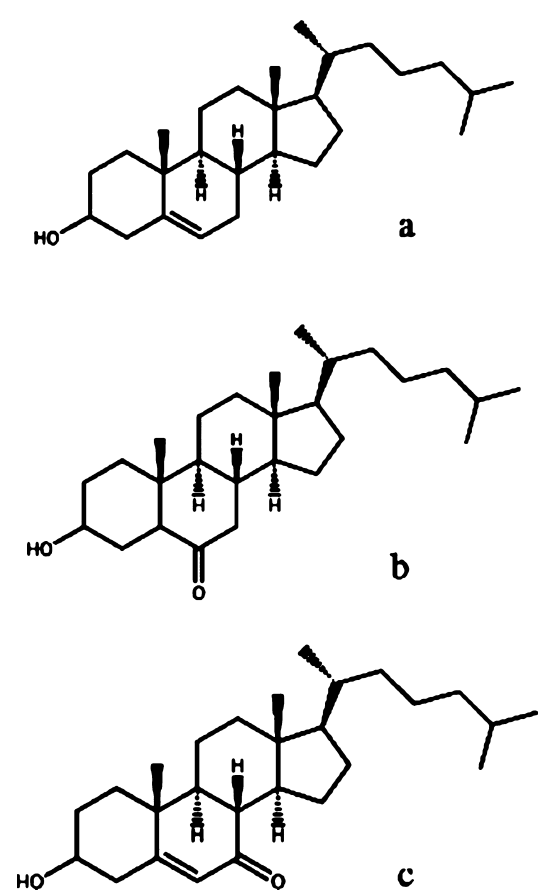

Figure 1. Chemical structures of cholesterol (a), 6-ketocholestanol (b), and 7-ketocholesterol (c).

ketocholestanol both increase $\psi_{\mathrm{d}}$ at sterol mole percentages up to the physiological level of cholesterol in the plasma membrane of around $40 \mathrm{~mol} \%$, but with the effect of 6 ketocholestanol being significantly greater than that of cholesterol. In contrast, 7-ketocholesterol causes a decrease in $\psi_{\mathrm{d}}$.

Experimentally it has been found that, on increasing the level of either cholesterol, 6-ketocholestanol or 7-ketocholesterol in the membrane, the maximum heat capacity of the main phase transition [as measured by differential scanning calorimetry (DSC)] decreases significantly, until, at sterol levels of $\geq \sim 30$ mol \%, the transition disappears completely. The pretransition already disappears even at lower sterol concentrations. These observations are consistent with the formation of a liquidordered phase, $l_{o}$, across the entire temperature range studied $\left(5-65{ }^{\circ} \mathrm{C}\right)$, as reported previously by other authors for mixtures of DMPC and cholesterol ${ }^{29,30}$ and the disappearance of the solid-ordered, $s_{o}$, (gel) and liquid-disordered, $1_{d}$, (liquid crystalline) phases.

More interesting is the comparison of the effects caused by cholesterol, 6-ketocholestanol, and 7-ketocholesterol (see Figure 2). In the case of 7-ketocholesterol, the sterol derivative which decreases $\psi_{\mathrm{d}}$, a prominent second peak appears. In the case of cholesterol, which increases $\psi_{\mathrm{d}}$, a second peak is also observed, but it is less pronounced than in the case of 7 ketocholesterol. In the experiments using 6-ketocholestanol, the derivative which causes the greatest increase in $\psi_{\mathrm{d}}$, rather than a clearly defined second peak, only a shoulder is observed, 


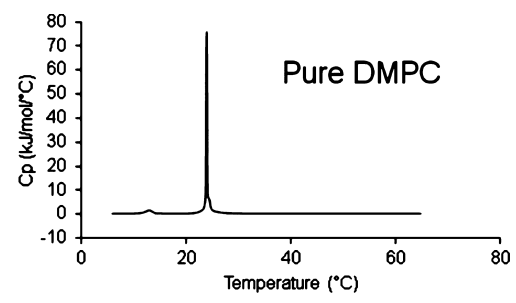

6-ketocholestanol
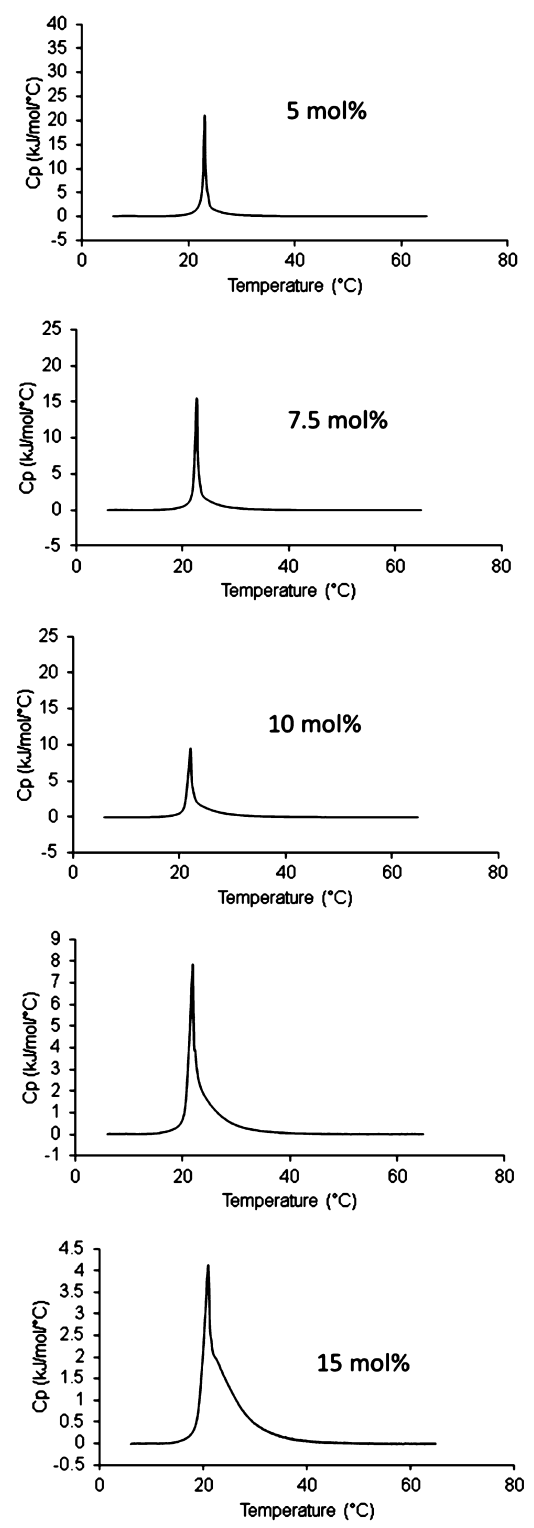

Cholesterol
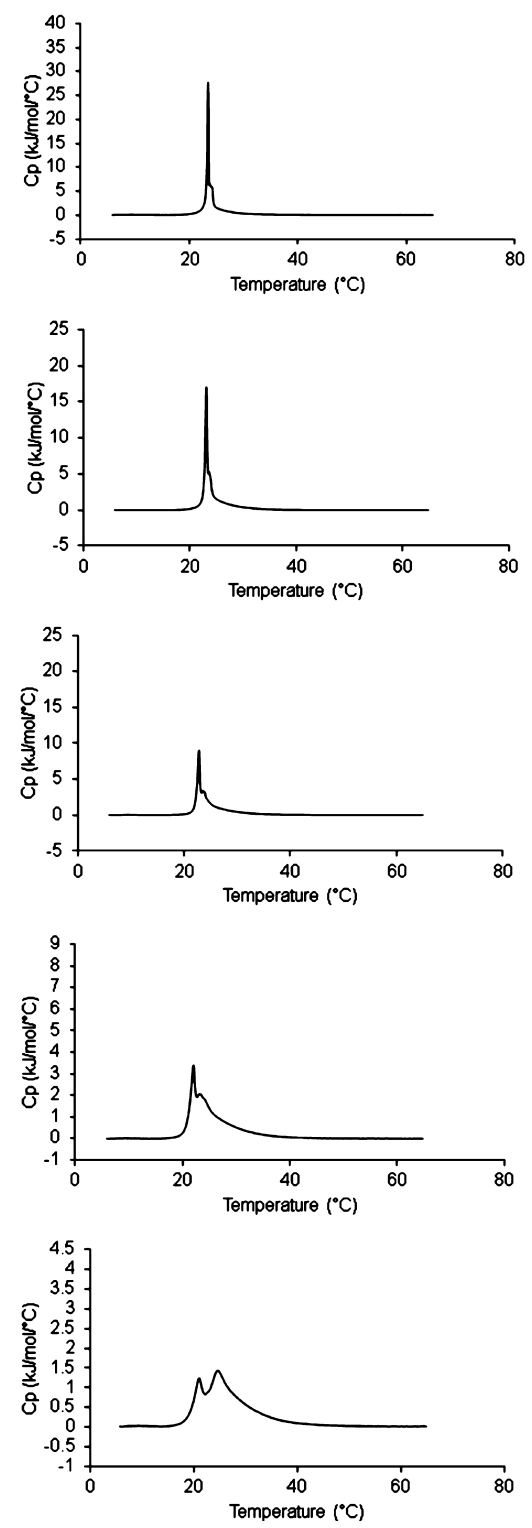

7-ketocholesterol
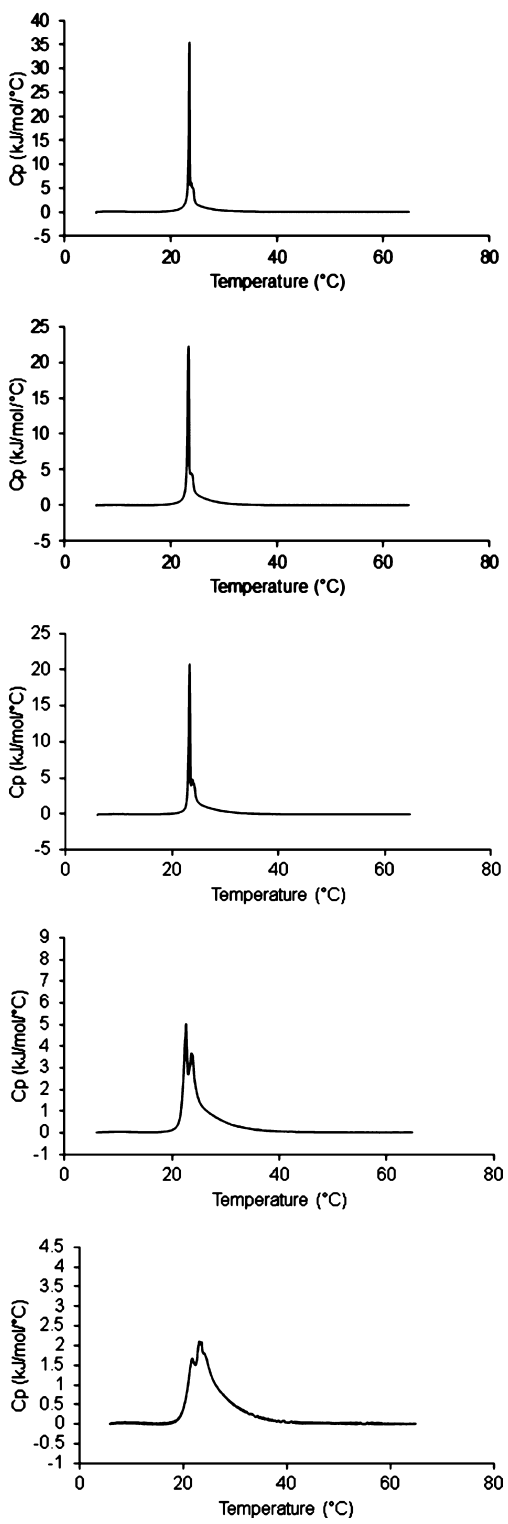

Figure 2. DSC Thermograms of DMPC multilamellar vesicles containing varying mole percentages of either 6-ketocholestanol, cholesterol, or 7ketocholesterol. See Tables S1-S3 for transition temperatures and enthalpy changes.

that is, the second peak is hidden by the more prominent peak on the low-temperature side of the transition. Thus, the prominence of the second peak appears to depend inversely on how strongly the sterol affects $\psi_{\mathrm{d}}$. Here, it is important to keep in mind that the effect of sterols on $\psi_{\mathrm{d}}$ arises not only from the component of their dipole moments perpendicular to the membrane surface but also from their effects on lipid packing and water penetration into the membrane, which would affect the local dielectric constant. ${ }^{28}$ It has been well established that sterols have a significant effect on lipid packing. ${ }^{31-33}$
The different effects of the sterol derivatives on the second peak or shoulder are consistent with its attribution to dipoledipole interactions (including water dipoles) in the lipid head group region. Sterols such as cholesterol and 6-ketocholestanol, which increase $\psi_{\mathrm{d}}$, would increase the membraneperpendicular dipole-dipole repulsion forces within the head group region, leading to a net decrease in the overall dipoledipole head group attraction. In contrast, 7-ketocholesterol, which decreases $\psi_{\mathrm{d}}$, would decrease the membrane-perpendicular dipole-dipole repulsion forces, allowing membrane in- 
plane dipole-dipole attraction to become more prominent, resulting in a net increase in overall dipole-dipole attraction. This appears to be reflected in the more prominent second peak observed in the measurements, where 7-ketocholesterol is added to the membranes.

Another possible interpretation of the second peak could be the separation of the membrane into sterol-rich and sterol-poor regions, with both regions having different strengths of van der Waals interactions between the lipid tails and, for this reason alone, having different phase transition temperatures. Indeed, phase separation of multicomponent membranes including cholesterol has been both theoretically predicted ${ }^{34}$ and experimentally observed. ${ }^{35}$ However, if in-plane dipole-dipole attraction were the cause for the second peak or shoulder in mixed DMPC/sterol membranes, then in principle, such an interaction should also be present in pure DMPC membranes. Therefore, we carried out further investigations on both pure DMPC and pure DPPC (dipalmitoylphosphatidylcholine) in order to determine whether evidence for electrostatic interactions in the head group region could be found.

Gel-to-Liquid-Crystalline Phase Transition of Pure DMPC Membranes. If the gel-to-liquid-crystalline phase transition of a lipid bilayer were due purely to a single cooperative process, that is, the cooperative breaking of van der Waals forces between the lipid hydrocarbon chains, then the heat capacity, $C_{p}$, versus temperature, $T$, profile of the membrane in the region of the phase transition should be described by a single symmetrical peak. Mathematically, the dependence of $C_{p}$ on $T$ for such a system is given by eq 7 (see the Experimental Methods).

Experimentally, however, it was found that it was impossible to fit the DSC curve of pure DMPC to eq 7. Instead, an equation describing the sum of three peaks was required (see Figure 3). Two of the peaks are relatively sharp (red and blue components in Figure 3), whereas the third (green) is very broad. To test whether any of the peaks could be due to components in the buffer solution interacting with the membrane, DSC scans were also performed for DMPC vesicles in solutions from which components of the buffer mixture were removed. In all cases, three peaks were necessary to adequately fit the data. Thus, the three peaks are not due to interaction of any buffer components with the membrane; they are an intrinsic property of the DMPC/water system, although modulated by the solution composition.

It was noticed that the main phase transition temperature shifted from $23.5{ }^{\circ} \mathrm{C}$ in buffer containing $30 \mathrm{mM}$ Tris, $150 \mathrm{mM}$ $\mathrm{NaCl}$, and $1 \mathrm{mM}$ EDTA at $\mathrm{pH} 7.2$ to $24.1{ }^{\circ} \mathrm{C}$ in pure water. From experiments in which $\mathrm{NaCl}$ was totally removed from the buffer, it could be shown that this shift is predominantly due to removal of salt from the solution. This can be explained by $\mathrm{NaCl}$ screening of polar interactions within the head group region of the membrane, which does not occur when the DMPC vesicles are prepared in pure water. At the $\mathrm{NaCl}$ concentration used, $150 \mathrm{mM}$, specific binding of either $\mathrm{Na}^{+}$or $\mathrm{Cl}^{-}$to the membrane surface appears to be very unlikely. Pabst et $\mathrm{al}^{36}$ have estimated that saturation of a PC membrane surface with $\mathrm{Na}^{+}$ions only occurs at salt concentrations $>6.7$ M. Chloride ions have been reported to bind to PC membranes only with a marginally higher affinity than $\mathrm{Na}^{+}{ }^{37}$

Another significant observation was that, after removal of $\mathrm{NaCl}$ from the buffer, an obvious shoulder appeared on the high-temperature side of the main transition peak (see Figure 3 ). Both shifts in the transition temperature and the change in
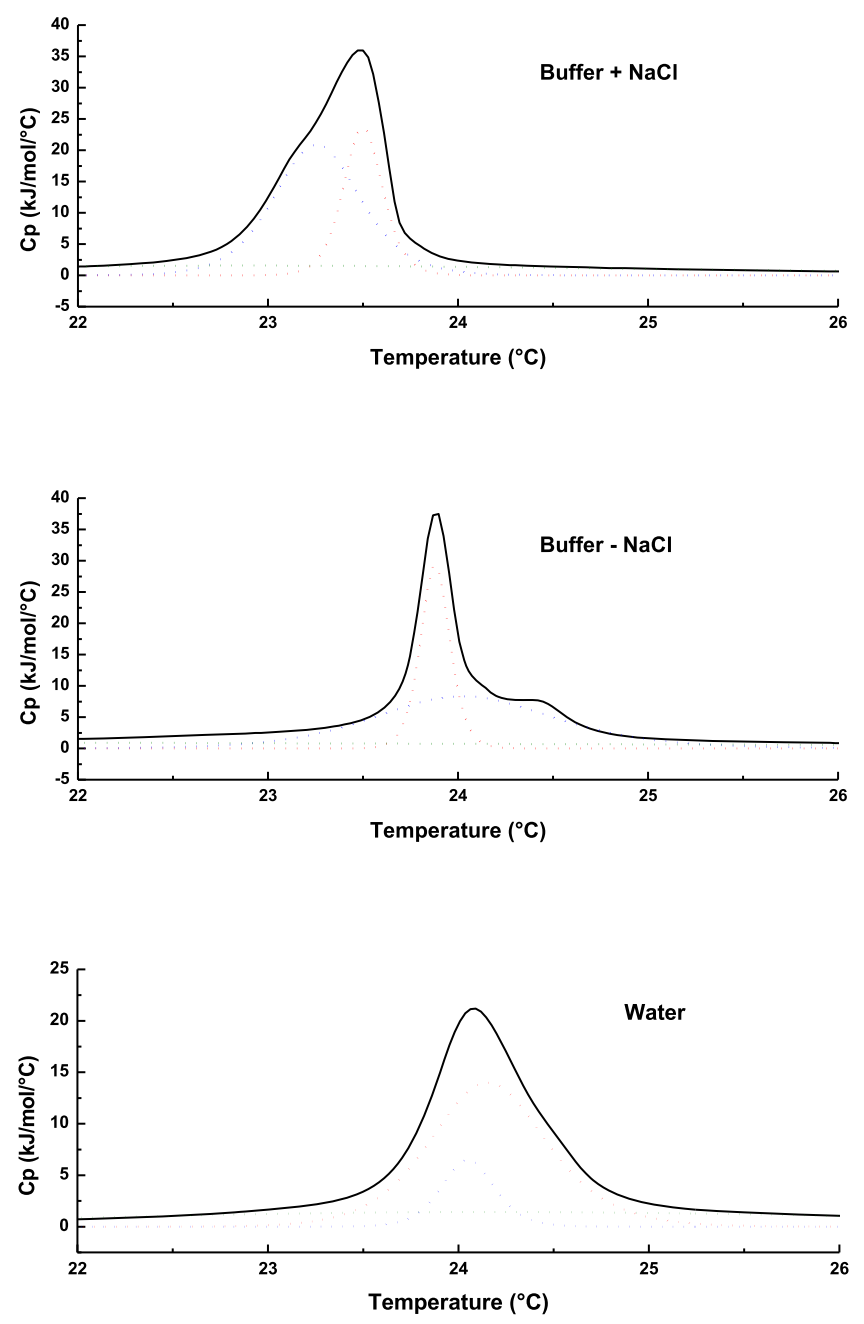

Figure 3. DSC profiles of DMPC in buffer containing $30 \mathrm{mM}$ Tris, $150 \mathrm{mM} \mathrm{NaCl}$, and $1 \mathrm{mM}$ EDTA adjusted to $\mathrm{pH} 7.2$ using $\mathrm{HCl}$ (top), in the same buffer but excluding $\mathrm{NaCl}$ (middle) and in water (bottom). The solid lines are the experimental data. The dotted curves are the deconvoluted components, obtained via fitting to a sum of 3 functions described by eq 7. Values of the fit parameters obtained are given in Table S4 of the Supporting Information. Averaging the total areas under the thermograms obtained in each of the three suspension media yields an average $\Delta H$ of $26( \pm 3) \mathrm{kJ} \mathrm{mol}^{-1}$, which is consistent with previous measurements. ${ }^{39}$

the shape of the main transition on $\mathrm{NaCl}$ removal are consistent with polar interactions between lipid head groups and their associated water molecules playing a significant role in determining the phase transition energetics. It is important to note that shifts of the phase transition temperature to lower values would be expected ${ }^{38}$ if a solute in the solution preferentially dissolved or interacted with lipid molecules in the liquid-crystalline phase and hence stabilized this state relative to the gel state (i.e., analogous to freezing point depression of ideal solutions). However, this analogy is strictly valid only if the membrane could be considered as an isotropic solvent and this is definitely not true. As described in the Introduction, a membrane is anisotropic, with polar and nonpolar regions to which solutes can bind with different affinities. Furthermore, the ideal solution analogy does not explain either the shoulder evident on $\mathrm{NaCl}$ removal or the three functions necessary to describe the experimental curve obtained in pure water. 
The results described above show that the gel-to-liquidcrystalline phase transition of DMPC is not simply due to the breaking of van der Waals forces between the lipid hydrocarbon chains. Other endothermic processes must also be involved. If the transition is not alone due to the hydrocarbon chains, the only other possible contribution would seem to be from the polar head group region, that is, dipole-dipole attractive forces in the head group region must also be overcome.

The alignment of dipoles perpendicular to the membrane surface, which gives rise to $\psi_{\mathrm{d}}$ must produce a repulsive interaction between neighboring lipid molecules. Therefore, this cannot account for the additional functions required to describe the DSC curve observed for pure DMPC. Thus, it would seem that the extra endothermic processes can only arise from dipolar components parallel to the membrane surface, such as the PC head group and associated water molecules. To test this conclusion further, we studied the phase behavior of DPPC membranes under the same experimental conditions as those used for DMPC. With its longer hydrocarbon tails, that is, 16 carbon atoms instead of 14 for DMPC, DPPC has stronger van der Waals forces between the hydrocarbon tails than DMPC. Because the head groups of DMPC and DPPC are identical, one would expect, however, no significant difference in polar forces within the head group region between the two lipids. Thus, the relative strength of van der Waals forces between the lipid tails to any polar forces within the head groups would increase on going from DMPC to DPPC.

Gel-to-Liquid-Crystalline Phase transition of Pure DPPC Membranes. Significant differences were observed between the DSC profiles of DPPC (see Figure 4) and those of DMPC (see Figure 2). First, as has been reported often before, ${ }^{40-44}$ the main phase transition of DPPC occurs at a temperature of $41.4{ }^{\circ} \mathrm{C}$, approximately $18^{\circ}$ higher than that of DMPC. This is clearly due to the longer hydrocarbon tails of DPPC and hence stronger attractive van der Waals forces between the tails. The phase transition of DPPC is also sharper, that is, more cooperative, than that of DMPC. This is also to be expected, because more van der Waals attractions need to be cooperatively broken in DPPC than in DMPC in order to convert the membrane from the gel to the liquidcrystalline state.

A similarity between the DPPC and DMPC curves is that more than one mathematical peak function was required to describe the observed data. This is particularly the case for DPPC in pure water, which displays a DSC thermogram which is significantly broader than that observed for DPPC in buffer. The fact that the DSC profile is much sharper in buffer than in water suggests that attractive polar interactions in the head group region are effectively screened in buffer solution. In contrast to DMPC, however, DPPC shows very little difference in the DSC behavior observed in buffers with and without $\mathrm{NaCl}$. Also in contrast to DMPC, in DPPC there is no observable difference in the phase transition temperature in the three different solutions, that is, the phase transition occurs at $41.4{ }^{\circ} \mathrm{C}$ in each case. Thus, it appears that the polar interactions between the PC head groups play a much smaller role in the energetics of the phase transition of DPPC compared to DMPC and that in DPPC, they are largely screened even before the addition of $\mathrm{NaCl}$ to the buffer. The observed results are, thus, consistent with strengthened van der
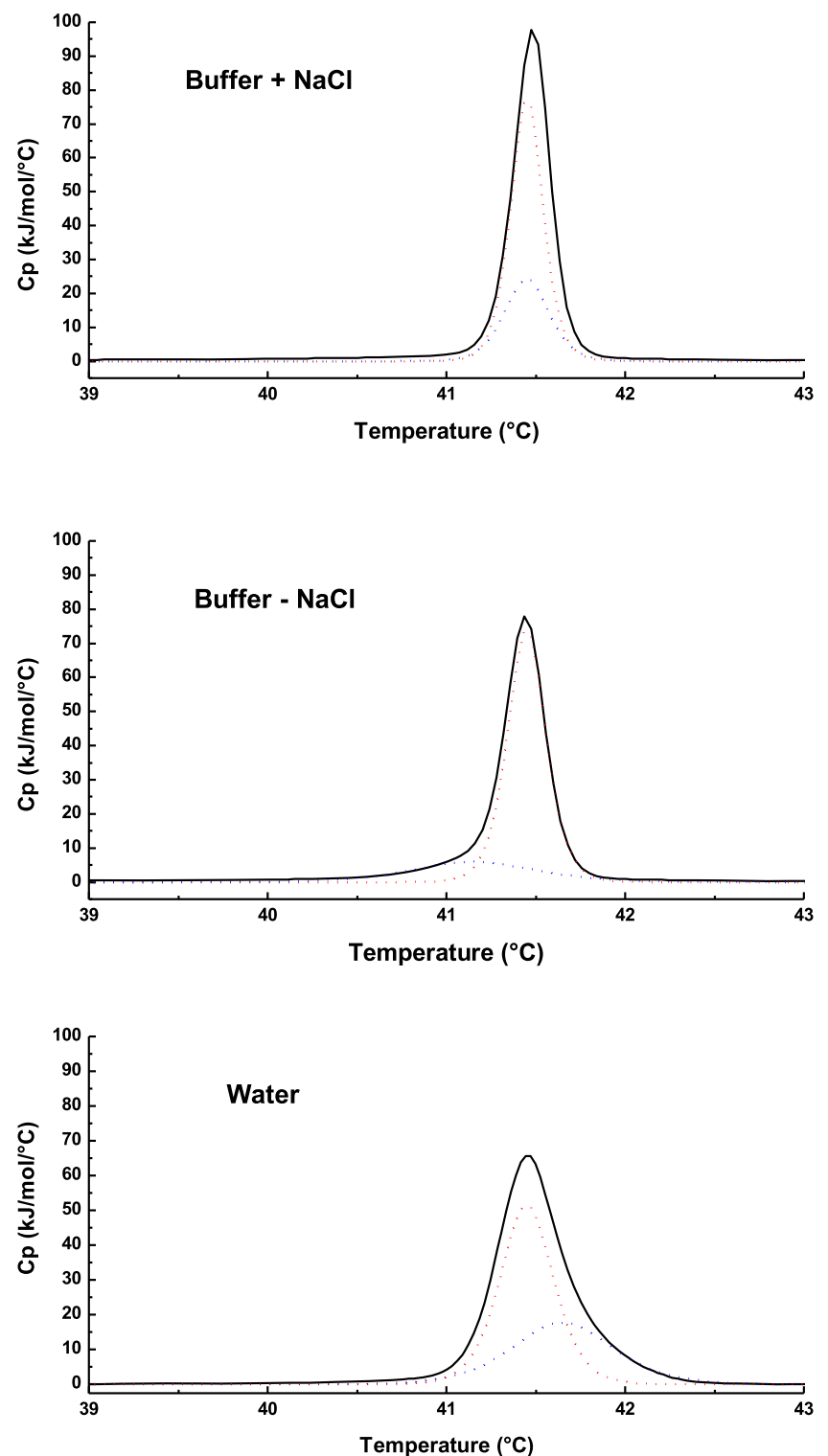

Figure 4. DSC profiles of DPPC in buffer containing $30 \mathrm{mM}$ Tris, $150 \mathrm{mM} \mathrm{NaCl}$, and $1 \mathrm{mM}$ EDTA adjusted to $\mathrm{pH} 7.2$ using $\mathrm{HCl}$ (top), in the same buffer but excluding $\mathrm{NaCl}$ (middle) and in water (bottom). The solid lines are the experimental data. The dotted curves are the deconvoluted components, obtained via fitting to a sum of two functions described by eq 7 . Values of the fit parameters obtained are given in Table S5 of the Supporting Information. Averaging the total areas under the thermograms obtained in each of the three suspension media yields an average $\Delta H$ of $29( \pm 4) \mathrm{kJ}$ $\mathrm{mol}^{-1}$, which is consistent with previous measurements. ${ }^{39}$

Waals forces relative to polar forces on going from DMPC to DPPC, as predicted in the previous section.

\section{DISCUSSION}

The DSC results described here on pure DMPC and DPPC with and without $\mathrm{NaCl}$ in the buffer and on DMPC membranes to which sterols have been added indicate that additional endothermic processes are involved in the lipid's main phase transition apart from the breaking of van der Waals forces between the lipid hydrocarbon tails. The fact that the energetics of the transition of DMPC are dependent on the presence or absence of salt in the surrounding buffer and that 
the salt effects are reduced in DPPC with stronger van der Waals forces between the lipid tails implicates a role of polar interactions in the transition. It is worthwhile noting that additional peaks or shoulders have previously been observed in DSC scans of shorter chain PCs (12 and 13 carbon atoms), but the physical basis for them was not determined. ${ }^{42,45}$

From integration of the area under the DSC curves, the total enthalpy change per mole of lipid for the breaking of both van der Waals interactions between the hydrocarbon chains and polar interactions between the lipid head groups and their associated water molecules can be experimentally obtained. It is interesting to see if the enthalpy change obtained for the shoulder of the DMPC transition is consistent with what one would expect from a theoretical point of view for a dipoledipole lipid head group interaction.

If the dipoles of the lipid head groups are aligned approximately in a colinear fashion, as experimental data indicate, ${ }^{10,11}$ the interaction of a pair of neighboring head groups would be attractive. Assuming the distance of separation of two dipoles is much greater than the distance between the two poles of the dipole, the energy, $E$, of interaction of two colinear dipoles in isolation is given by ${ }^{46}$

$$
E=-\frac{1}{4 \pi \varepsilon_{0} \varepsilon} \frac{2 \mu^{2}}{r^{3}}
$$

where $\mu$ is the dipole moment, $r$ is the distance between the two dipoles, $\varepsilon_{0}$ is the permittivity of free space, and $\varepsilon$ is the dielectric constant of the medium. In a two-dimensional array of dipoles, as present in a membrane surface, the magnitude of the attractive energy would be modulated by a factor $M$ because of interactions with all neighboring dipoles as well as those more distant, where $M$ is the Madelung constant determined by the geometric packing of the dipoles.

The dipole moment of the PC head group has been estimated from dielectric dispersion measurements to have a value of $19 \mathrm{D}$ or $6.4 \times 10^{-29} \mathrm{C} \mathrm{m} .^{47}$ In the gel phase, the area occupied per lipid in the plane of the membrane has been determined to be $49 \AA^{2}$, ${ }^{48}$ so that the maximum possible separation between two neighboring head group dipoles is $7 \AA$. Using these values for $\mu$ and $r$ in eq 1 and assuming a dielectric constant close to water of 80 yields a lower limit of the energy of interaction between two isolated lipid head group dipoles of $1.6 \mathrm{~kJ} \mathrm{~mol}^{-1}$. However, theoretical calculations have indicated that the dielectric constant at the position of the PC head group could in fact be much greater than that of pure water, and a value of 210 has been estimated. ${ }^{49}$ Based on this value, the energy of interaction of two isolated head group dipoles would only be expected to be $0.015 \mathrm{~kJ} \mathrm{~mol}^{-1}$.

To estimate a molar energy of interaction for the extended lattice of the membrane and take into account both nearest and non-nearest neighbor interactions, the value of $0.015 \mathrm{~kJ}$ $\mathrm{mol}^{-1}$ would need to be multiplied by the appropriate value of $M$ for the lattice. Typically, Madelung constants are in the range $1-4,50$ but even taking an extreme value of 10 , only results in an interaction energy of around $0.15 \mathrm{~kJ} \mathrm{~mol}^{-1}$. Comparing this value to the experimentally determined total enthalpy change for a gel-to-liquid-crystalline phase transition of DMPC of $23-29 \mathrm{~kJ} \mathrm{~mol}^{-1}$, it indicates that, from a theoretical point of view, dipole-dipole interactions between PC dipoles of the head group in the plane of the membrane would not be expected to make a major contribution to the overall energetics of the transition.
However, as described in the Introduction, the PC head groups are thought to be hydrogen bonded to hydrating water molecules, forming a hydrogen-bonded water network across the membrane surface. ${ }^{16,20}$ Therefore, rather than the additional peak in the DSC profile of DMPC being associated with disruption of dipole-dipole forces between PC dipoles, it seems more likely that it is due to disruption of the hydrogenbonded water network. This would be consistent with the infrared spectroscopic measurements of Arrondo et al., ${ }^{17}$ showing that the gel-to-liquid-crystalline phase transition causes no change in the wavenumbers of phosphate vibrations in the range of $1000-1300 \mathrm{~cm}^{-1}$. For a comparison of the energy change expected on partial disruption of a hydrogenbonded water network, one could consider the enthalpy change associated with the melting of ice into liquid water, that is, the enthalpy of $\mathrm{H}_{2} \mathrm{O}$ fusion, which has a value of $6.00 \mathrm{~kJ}$ $\mathrm{mol}^{-1} .51$ This value is approximately one-quarter of the total enthalpy change associated with the main phase transition of DMPC. Of course, the enthalpy of $\mathrm{H}_{2} \mathrm{O}$ fusion is given per mole of water molecules, whereas the enthalpy change associated with the main phase transition of DMPC is given per mole of lipid molecules. However, the number of water molecules per lipid molecule is not expected to be large. X-ray crystal structural analysis shows two waters per lipid. ${ }^{16}$ Thus, it seems likely that the additional process associated with the gelto-liquid-crystalline transition of DMPC which is responsible for the shoulder or extra peak on the high-temperature side of the transition is due to the disruption of the hydrogen-bonded water network at the membrane surface.

Because DSC is not a structural technique, it is worth considering recent spectroscopic data which are consistent with the interpretation presented here. The orientation and dynamics of water interacting with surfaces, such as biological membranes, can now be investigated by techniques of vibrational sum frequency generation (VSFG) spectroscopy. ${ }^{52-55}$ Using heterodyne-detected VSFG spectroscopy, Mondal et al. ${ }^{53}$ distinguished three distinct water structures at a PC/water interface: (1) water associated with the negatively charged phosphate $\left(\mathrm{W}_{\mathrm{P}}\right)$, with the positive hydrogen end of the water dipole pointing toward the phosphate; (2) water associated with the positively charged choline $\left(\mathrm{W}_{\mathrm{C}}\right)$, with the negative oxygen end of the water dipole pointing toward the nitrogen atom of the choline; and (3) water interacting with the lipid at the level of its glycerol backbone $\left(\mathrm{W}_{\mathrm{H}}\right)$, with the positive hydrogen end of the water dipole pointing toward the ester linkage to the hydrocarbon chains. Because of their location, in a region of lower dielectric constant, and their orientation, it would seem likely that the $\mathrm{W}_{\mathrm{H}}$ water molecules could be a major contributor to the inside-positive $\psi_{\mathrm{d}}$. This would be consistent with previous reports suggesting a major contribution from water in this location to the sign and magnitude of $\psi_{\mathrm{d}} \cdot{ }^{56-59}$ The results of Gawrisch et al. ${ }^{57}$ and those of Starke-Peterkovic and Clarke ${ }^{59}$ in particular indicate that water most likely interacts with the carbonyl group of the sn-2 hydrocarbon chain.

The $\mathrm{W}_{\mathrm{P}}$ and $\mathrm{W}_{\mathrm{C}}$ waters, on the other hand, are more likely to be involved in a hydrogen-bonded water network, which we propose here is disrupted by the main phase transition of the lipid. Via time-resolved heterodyne-detected VSFG measurements, Inoue et al. ${ }^{20}$ showed that the dynamics of hydrogen bond fluctuation of the $\mathrm{W}_{\mathrm{P}}$ waters is influenced by that of the $\mathrm{W}_{\mathrm{C}}$ waters. Thus, there exists cooperativity in hydrogen bonding extending across the membrane surface. It is 
important to note that all of the structural information about the zwitterionic PC/water surface gained via VSFG spectroscopy has been obtained via studies on lipid monolayers spread on a water/air interface. The results obtained here using DSC on lipid vesicles not only provide thermodynamic information on the strength of attractive polar interactions at the DMPC membrane surface but they also provide evidence that the structural and dynamic information obtained by VSFG spectroscopy hold true for the more biologically relevant lipid bilayer system. On the basis of the thermodynamic data obtained here via DSC, the structural information from VSFG, and spectroscopic data on the dipole potential, it is possible to construct the schematic diagram of the hydration of $\mathrm{PC} /$ water interface shown in Figure 5.

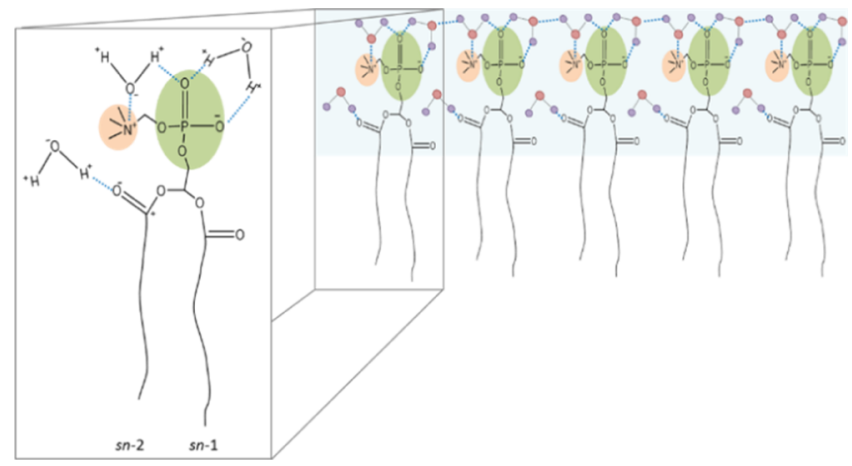

Figure 5. Schematic diagram of the surface of a PC membrane. $\mathrm{H}_{2} \mathrm{O}$ molecules hydrogen bonded to the carbonyl group of the sn-2 hydrocarbon chain are thought to be a major contributor to the inside-positive dipole potential, $\psi_{\mathrm{d}}$, which causes a membraneperpendicular polar repulsion between neighboring lipid molecules. $\mathrm{H}_{2} \mathrm{O}$ dipoles on the membrane surface are shown in alternating hydrogens-up (H-bonded to the positive choline residue) and hydrogens-down (H-bonded to the negative phosphate group) orientations, which via $\mathrm{H}$ bonding with each other form an attractive in-plane $\mathrm{H}$-bonded $\mathrm{H}_{2} \mathrm{O}$ network across the surface. In the expanded lipid molecule, partial charges on the $\mathrm{H}_{2} \mathrm{O}$ molecules and the sn-2 chains carbonyl group are shown, in addition to full charges on the choline and phosphate groups. The carbonyl group of the sn-2 chain is thought to be oriented at an angle, with its oxygen pointing toward the membrane surface, thus orienting $\mathrm{H}_{2} \mathrm{O}$ molecules with their hydrogens pointing toward the membrane interior. ${ }^{57,59}$

\section{CONCLUSIONS}

Finally, returning to the initial motivation for this study, in the light of the new results presented here, it is important to consider whether local deformations in head group packing of lipids caused by conformational changes of membrane proteins which alter the local membrane hydrophobic thickness could make a significant contribution to the relative thermodynamic stability of different protein conformational states, as previously proposed. ${ }^{9}$ The DSC results have shown that enthalpy changes associated with changes in head group hydration become more dominant relative to van der Waals interactions between the lipid hydrocarbon tails when the hydrocarbon tails become shorter or when their interaction is weakened by the introduction of cholesterol or another sterol. In the biological membranes of animals, van der Waals interactions between the lipid hydrocarbon tails are expected to be much weaker than, for example, in DPPC, not only because of the presence of cholesterol (up to $40 \mathrm{~mol} \%$ ) but also because of the presence of lipids with hydrocarbon chains with significant degrees of cis-unsaturation, which drastically perturbs chain packing. Under these circumstances, one would, therefore, expect the contribution of lipid head group hydration to the thermodynamics of membrane structural changes to be even more important than that observed here for the phase transition of DMPC. Previously, however, only the repulsive membrane-perpendicular component to the energetics of membrane bending arising from the dipole potential was considered. ${ }^{9}$ The DSC results presented here indicate that an attractive in-plane interaction in the head group region would also be involved. Thus, the energy cost in local outward bending of a membrane to accommodate an increase in hydrophobic thickness of a membrane protein arising from an increase in the local dipole potential (and an increase in lipid packing density) is likely to be reduced by an energetically favorable increase in the in-plane polar attraction.

\section{EXPERIMENTAL METHODS}

Materials. Origins of the reagents used were as follows: DMPC (Avanti Polar Lipids, Alabaster, AL, USA), ethylenediamine tetraacetic acid (EDTA, $\geq 99 \%$, Sigma, Castle Hill, Australia), tris[hydroxymethyl] aminomethane (Tris, 99\%, Alfa Aesar, Heysham, UK), sodium chloride (analytical grade, Merck, Kilsyth, Australia), $\mathrm{HCl}$ (0.1 N Titrisol solution, Merck), and chloroform ( $\geq 99.0 \%$, Uvasol, Merck). Purified water (18.2 M $\Omega$ ) was obtained via a Millipore Direct-Q 5UVR system (Merck Millipore, Bayswater, Australia).

Vesicle Preparation. Separate stock solutions of DMPC and of each sterol used were first prepared at a concentration of $3 \mathrm{mM}$ in chloroform. All solutions were prepared by weight, using a chloroform density of $1.48 \mathrm{~g} \mathrm{~mL}^{-1}$. A $5 \mathrm{~mL}$ sample of the DMPC stock was then mixed with the appropriate volume of the sterol stock to obtain the desired mole percentages in the vesicles subsequently formed (e.g., for a preparation of 20 $\mathrm{mol} \%$ sterol, $5 \mathrm{~mL}$ of DMPC and $1.25 \mathrm{~mL}$ of sterol stock were mixed). The chloroform was then removed from each sample via rotary evaporation under vacuum for 30-40 $\mathrm{min}$ at maximum rotation speed using R-114 Rotavapor (Büchi, Flawil, Switzerland) to form a thin lipid film on the walls of a round-bottom flask. The vacuum was maintained initially at 474 mbar using a V-850 vacuum controller (Büchi). After no visible traces of chloroform could be detected in the flask, the resulting film was dried for a further $30 \mathrm{~min}$ at $10 \mathrm{mbar}$. Throughout rotary evaporation, the thermal bath was kept at a temperature of approximately $40{ }^{\circ} \mathrm{C}$. Multilamellar vesicles were then formed by resuspending the lipid film in $5 \mathrm{~mL}$ of either water or buffer containing $30 \mathrm{mM}$ Tris, $150 \mathrm{mM} \mathrm{NaCl}$, and $1 \mathrm{mM}$ EDTA (adjusted to $\mathrm{pH} 7.2$ using $\mathrm{HCl}$ ). Via this procedure, the molar concentration of DMPC in the DSC cell is always $3 \mathrm{mM}$ for every scan, regardless of the mole percentage of sterol. Furthermore, because the sterols are present with the phospholipid in chloroform solution before rotary evaporation and resuspension in aqueous solution, it can be assumed that the sterols are distributed through all leaflets of the multilamellar vesicles formed.

Differential Scanning Calorimetry. DSC measurements were performed using a VP-DSC microcalorimeter (Microcal, Northampton, MA). Prior to analysis of a sample, seven scans were collected and averaged. The baseline, obtained using the appropriate suspension medium, that is, buffer or water, in both the sample and reference cells of the instrument, was then subtracted from the sample scan. To avoid scan rate-dependent 
shifts in the observed phase transitions, low-temperature scan rates of either 10 or $5^{\circ} \mathrm{C} \mathrm{h}^{-1}$ were used for all measurements. The lower scan rate of $5{ }^{\circ} \mathrm{C} \mathrm{h}^{-1}$ was used for measurements of DPPC in buffer with and without $\mathrm{NaCl}$, because in these cases, the main phase transitions occur over a particularly small temperature range. This allowed the collection of more data points, thus assisting in deconvolution of the measured DSC curves.

Data Fitting. If the gel-to-liquid-crystalline phase transition of a lipid bilayer were due purely to a single cooperative process, that is, the cooperative breaking of van der Waals forces between the lipid hydrocarbon chains, then the heat capacity, $C_{p}$, versus temperature, $T$, profile of the membrane in the region of the phase transition (as measured by DSC) should be described by a single symmetrical peak. Mathematically, the dependence of $C_{p}$ on $T$ for such a system is given by eq 2

$$
C_{p}=\frac{\exp \left\{-\frac{N_{0} \Delta H}{R T}\left(1-\frac{T}{T_{\mathrm{m}}}\right)\right\}}{\left[1+\exp \left\{-\frac{N_{0} \Delta H}{R T}\left(1-\frac{T}{T_{\mathrm{m}}}\right)\right\}\right]^{2}} \cdot \frac{N_{0} \Delta H^{2}}{R T^{2}}
$$

where $R$ is the universal gas constant, $N_{0}$ is the size of the cooperative unit (i.e., the number of lipid molecules cooperatively undergoing the transition), $T_{\mathrm{m}}$ is the phase transition temperature (i.e., the midpoint temperature of the transition), and $\Delta H$ is the enthalpy change per mole of lipid for the entire transition. $\Delta H$ is given by the integral of $C_{p}$ with respect to $T$.

The derivation of eq 2 is based on the following three basic equations

$$
\begin{aligned}
& C_{p}=\Delta H \cdot \frac{\mathrm{d} x}{\mathrm{~d} T} \\
& \frac{\mathrm{d} x}{\mathrm{~d} T}=\frac{K}{(1+K)^{2}} \cdot \frac{N_{0} \Delta H}{R T^{2}} \\
& K=\exp \left\{-\frac{N_{0} \Delta H}{R T}\left(1-\frac{T}{T_{\mathrm{m}}}\right)\right\}
\end{aligned}
$$

where $x$ is the degree of the transition, that is, in our case considering just the gel-to-liquid-crystalline phase transition $x$ $=0$ in the gel phase at temperatures far below $T_{\mathrm{m}}$ and $x=1$ in the liquid-crystalline phase at temperatures far above $T_{\mathrm{m}}$. Equation 3 is simply the mathematical definition of heat capacity at constant pressure, that is, the differential of the heat transferred with respect to temperature. Equation 4 is derived from the van't Hoff equation for the dependence of the equilibrium constant of a reaction on temperature. Equation 5 is derived from the relationship between $K$ and the standard Gibbs free energy change, $\Delta G^{0}$, which, by definition must be zero at $T=T_{\mathrm{m}}$ (where $\left.K=1\right)$, that is $K=\exp \left(-\Delta G^{0} / R T\right)$. Expanding $\Delta G^{0}$ into entropy and enthalpy components, the entropy component can be eliminated by expressing it in the form $\Delta S^{0}=\Delta H^{0} / T_{\mathrm{m}}$, where $\Delta H^{0}$ is the van't Hoff enthalpy change for the transition $\left(=N_{0} \Delta H\right)$.

Nonlinear regression fitting of experimental data was carried out using the commercially available program Prism 7.02 (GraphPad Software Inc., La Jolla, CA). Rather than fitting the experimental data directly to a sum of functions described by eq 2 , the size of the cooperative unit, $N_{0}$, was substituted by

$$
N_{0}=\frac{4 C_{p}^{\max } R T_{\mathrm{m}}{ }^{2}}{\Delta H^{2}}
$$

where $C_{p}^{\max }$ is the maximum value of the heat capacity at the phase transition temperature, $T_{\mathrm{m}}$. This yields the following fit function instead

$$
C_{p}=\frac{\exp \left\{-\frac{4 C_{p}^{\max } T_{\mathrm{m}}{ }^{2}}{\Delta H T}\left(1-\frac{T}{T_{\mathrm{m}}}\right)\right\}}{\left[1+\exp \left\{-\frac{4 C_{p}^{\max } T_{\mathrm{m}}{ }^{2}}{\Delta H T}\left(1-\frac{T}{T_{\mathrm{m}}}\right)\right\}\right]^{2}} \cdot \frac{4 C_{p}^{\max } T_{\mathrm{m}}{ }^{2}}{T^{2}}
$$

Although eqs 2 and 7 are mathematically identical, fitting to eq 7 has the advantage that an initial estimate of $C_{p}^{\max }$ can more easily be made from the experimental data than in the case of $N_{0}$. This improves the efficiency of the nonlinear fitting procedure. The fitting parameters were, thus, $T_{\mathrm{m}}, C_{p}^{\max }$, and $\Delta H$. Because $\Delta H$ is the integral of the heat capacity across an entire phase transition, for the purposes of fitting, initial estimates of $\Delta H$ for each component peak of a DSC curve were set to the total area under the curve.

\section{ASSOCIATED CONTENT}

\section{Supporting Information}

The Supporting Information is available free of charge on the ACS Publications website at DOI: 10.1021/acsomega. 8 b03102.

Analysis of DSC thermograms of DMPC containing varying mole percentages of cholesterol; analysis of DSC thermograms of DMPC containing varying mole percentages of 6-ketocholestanol; analysis of DSC thermograms of DMPC containing varying mole percentages of 7-ketocholesterol; analysis of DSC thermograms of pure DMPC under different solution conditions; and analysis of DSC thermograms of pure DPPC under different solution conditions (PDF) (PDF)

\section{AUTHOR INFORMATION}

\section{Corresponding Author}

*E-mail: ronald.clarke@sydney.edu.au. Phone: +61 29351 4406 (R.J.C.).

ORCID

Ronald J. Clarke: 0000-0002-0950-8017

Present Address

${ }^{\S}$ University of Technology Sydney, Sydney NSW 2007, Australia.

\section{Notes}

The authors declare no competing financial interest.

\section{ACKNOWLEDGMENTS}

The authors thank Assoc. Prof. James Beattie, Prof. Cameron Kepert, University of Sydney, and Prof. Toby Allen, RMIT University and Department of Chemistry, UC Davis, for helpful discussions. This work was funded by the Australian Research Council (Discovery grants nos. DP121003548, DP150101112, and DP170101732).

\section{REFERENCES}

(1) Brockman, H. Dipole potential of lipid membranes. Chem. Phys. Lipids 1994, 73, 57-79. 
(2) Clarke, R. J. The Dipole Potential of Phospholipid Membranes and Methods for its Detection. Adv. Colloid Interface Sci. 2001, 89-90, 263-281.

(3) O'Shea, P. Intermolecular Interactions with/within Cell Membranes and the Trinity of Membrane Potentials: Kinetics and Imaging. Biochem. Soc. Trans. 2003, 31, 990-996.

(4) Wang, L. Measurements and Implications of the Membrane Dipole Potential. Annu. Rev. Biochem. 2012, 81, 615-635.

(5) Jordan, P. C. Electrostatic modeling of ion pores. II. Effects attributable to the membrane dipole potential. Biophys. J. 1983, 41, 189-195.

(6) Mares, L. J.; Garcia, A.; Rasmussen, H. H.; Cornelius, F.; Mahmmoud, Y. A.; Berlin, J. R.; Lev, B.; Allen, T. W.; Clarke, R. J. Identification of Electric-Field-Dependent Steps in the $\mathrm{Na}^{+}, \mathrm{K}^{+}$-Pump Cycle. Biophys. J. 2014, 107, 1352-1363.

(7) Ganea, C.; Babes, A.; Lüpfert, C.; Grell, E.; Fendler, K.; Clarke, R. J. Hofmeister Effects of Anions on the Kinetics of Partial Reactions of the $\mathrm{Na}^{+}, \mathrm{K}^{+}$-ATPase. Biophys. J. 1999, 77, 267-281.

(8) Pearlstein, R. A.; Dickson, C. J.; Hornak, V. Contributions of the Membrane Dipole Potential to the Function of Voltage-Gated Cation Channels and Modulation by Small Molecule Potentiators. Biochim. Biophys. Acta, Biomembr. 2017, 1859, 177-194.

(9) Clarke, R. J. Dipole-Potential-Mediated Effects on Ion Pump Kinetics. Biophys. J. 2015, 109, 1513-1520.

(10) Yeagle, P. L. Phospholipid headgroup behavior in biological assemblies. Acc. Chem. Res. 1978, 11, 321-327.

(11) Büldt, G.; Gally, H. U.; Seelig, J.; Zaccai, G. Neutron Diffraction Studies on Phosphatidylcholine Model Membranes. J. Mol. Biol. 1979, 134, 673-691.

(12) Seelig, J.; MacDonald, P. M.; Scherer, P. G. Phospholipid Head Groups as Sensors of Electric Charge in Membranes. Biochemistry 1987, 26, 7535-7541.

(13) Bechinger, B.; Seelig, J. Conformational changes of the phosphatidylcholine headgroup due to membrane dehydration. A 2H-NMR study. Chem. Phys. Lipids 1991, 58, 1-5.

(14) Ulrich, A. S.; Watts, A. Molecular response of the lipid headgroup to bilayer hydration monitored by $2 \mathrm{H}-\mathrm{NMR}$. Biophys. J. 1994, 66, 1441-1449.

(15) Disalvo, E. A.; Lairion, F.; Martini, F.; Tymczyszyn, E.; Frías, M.; Almaleck, H.; Gordillo, G. J. Structural and Functional Properties of Hydration and Confined Water in Membrane Interfaces. Biochim. Biophys. Acta, Biomembr. 2008, 1778, 2655-2670.

(16) Hauser, H.; Pascher, I.; Pearson, R. H.; Sundell, S. Preferred Conformation and Molecular Packing of Phosphatidylethanolamine and Phosphatidylcholine. Biochim. Biophys. Acta, Rev. Biomembr. 1981, $650,21-51$.

(17) Arrondo, J. L. R.; Goñi, F. M.; Macarulla, J. M. Infrared spectroscopy of phosphatidylcholines in aqueous suspension a study of the phosphate group vibrations. Biochim. Biophys. Acta, Lipids Lipid Metab. 1984, 794, 165-168.

(18) Goñi, F. M.; Arrondo, J. L. R. A Study of Phospholipid Phosphate Groups in Model Membranes by Fourier Transform Infrared Spectroscopy. Faraday Discuss. Chem. Soc. 1986, 81, 117126.

(19) Pohle, W.; Selle, C.; Fritzsche, H.; Bohl, M. Comparative FTIR Spectroscopic Study upon the Hydration of Lecithins and Cephalins. J. Mol. Struct. 1997, 408-409, 273-277.

(20) Inoue, K.-i.; Singh, P. C.; Nihonyanagi, S.; Yamaguchi, S.; Tahara, T. Cooperative Hydrogen-bond Dynamics at a Zwitterionic Lipid/Water Interface Revealed by 2D HD-VSFG Spectroscopy. J. Phys. Chem. Lett. 2017, 8, 5160-5165.

(21) Janiak, M. J.; Small, D. M.; Shipley, G. G. Nature of the Thermal Pretransition of Synthetic Phospholipids: Dimyristoyl- and Dipalmitoyllecithin. Biochemistry 1976, 15, 4575-4580.

(22) Peschke, J.; Riegler, J.; Möhwald, H. Quantitative Analysis of Membrane Distortions Induced by Mismatch of Protein and Lipid Hydrophobic Thickness. Eur. Biophys. J. 1987, 14, 385-391.
(23) Killian, J. A. Hydrophobic Mismatch between Proteins and Lipids in Membranes. Biochim. Biophys. Acta Rev. Biomembr. 1998, 1376, 401-416.

(24) Lewis, R. N. A. H.; McElhaney, R. N. The Mesomorphic Phase Behavior of Lipid Bilayers. In The Structure of Biological Membranes, 2nd ed.; Yeagle, P. L., Ed.; CRC Press: Boca Raton, FL, 2005; pp 53120.

(25) Lewis, R. N. A. H.; McElhaney, R. N. Membrane Lipid Phase Transitions and Phase Organization Studied by Fourier Transform Infrared Spectroscopy. Biochim. Biophys. Acta, Biomembr. 2013, 1828, 2347-2358

(26) Gross, E.; Bedlack, R. S.; Loew, L. M. Dual-Wavelength Ratiometric Fluorescence Measurement of the Membrane Dipole Potential. Biophys. J. 1994, 67, 208-216.

(27) Clarke, R. J.; Kane, D. J. Optical Detection of Membrane Dipole Potential: Avoidance of Fluidity and Dye-Induced Effects. Biochim. Biophys. Acta, Biomembr. 1997, 1323, 223-239.

(28) Starke-Peterkovic, T.; Turner, N.; Vitha, M. F.; Waller, M. P.; Hibbs, D. E.; Clarke, R. J. Cholesterol Effect on the Dipole Potential of Lipid Membranes. Biophys. J. 2006, 90, 4060-4070.

(29) Mainali, L.; Raguz, M.; Subczynski, W. K. Formation of Cholesterol Bilayer Domains Precedes Formation of Cholesterol Crystals in Cholesterol/Dimyristoylphosphatidylcholine Membranes: EPR and DSC Studies. J. Phys. Chem. B 2013, 117, 8994-9003.

(30) Subczynski, W. K.; Pasenkiewicz-Gierula, M.; Widomska, J.; Mainali, L.; Raguz, M. High Cholesterol/Low Cholesterol: Effects in Biological Membranes: A Review. Cell Biochem. Biophys. 2017, 75, 369-385.

(31) Needham, D.; McIntosh, T. J.; Evans, E. Thermomechanical and Transition Properties of Dimyristoylphosphatidylcholine/Cholesterol Bilayers. Biochemistry 1988, 27, 4668-4673.

(32) Hung, W.-C.; Lee, M.-T.; Chen, F.-Y.; Huang, H. W. The Condensing Effect of Cholesterol in Lipid Bilayers. Biophys. J. 2007, 92, 3960-3967.

(33) Mills, T.; Huang, J.; Feigenson, G.; Nagle, J. Effects of Cholesterol and Unsaturated DOPC Lipid on Chain Packing of Saturated Gel-Phase DPPC Bilayers. Gen. Physiol. Biophys. 2009, 28, $126-139$.

(34) Galimzyanov, T. R.; Akimov, S. A. Phase separation in lipid membranes induced by the elastic properties of components. JETP Lett. 2011, 93, 463-469.

(35) Staneva, G.; Osipenko, D. S.; Galimzyanov, T. R.; Pavlov, K. V.; Akimov, S. A. Metabolic Precursor of Cholesterol Causes Formation of Chained Aggregates of Liquid-Ordered Domains. Langmuir 2016, 32, 1591-1600.

(36) Pabst, G.; Hodzic, A.; Štrancar, J.; Danner, S.; Rappolt, M.; Laggner, P. Rigidification of Neutral Lipid Bilayers in the Presence of Salts. Biophys. J. 2007, 93, 2688-2696.

(37) Tatulian, S. A. Binding of alkaline-earth metal cations and some anions to hosphatidylcholine liposomes. Eur. J. Biochem. 1987, 170, 413-420.

(38) Sturtevant, J. M. The Effects of Water-Soluble Solutes on the Phase Transitions of Phospholipids. Proc. Natl. Acad. Sci. U.S.A. 1984, 81, 1398-1400.

(39) Marsh, D. Handbook of Lipid Bilayers; CRC Press: Boca Raton, FL, 1990; pp 139-140.

(40) Jain, M. K.; Wu, N. M. Effect of small molecules on the dipalmitoyl lecithin liposomal bilayer: III. Phase transition in lipid bilayer. J. Membr. Biol. 1977, 34, 157-201.

(41) Mabrey, S.; Mateo, P. L.; Sturtevant, J. M. High-Sensitivity Scanning Calorimetric Study of Mixtures of Cholesterol with Dimyristoyl- and Dipalmitoylphosphatidylcholines. Biochemistry 1978, 17, 2464-2468.

(42) Blume, A. Applications of calorimetry to lipid model membranes. In Physical Properties of Biological Membranes and Their Functional Implications; Hidalgo, C., Ed.; Plenum: New York, 1988; pp $71-121$

(43) McMullen, T. P. W.; Lewis, R. N. A. H.; McElhaney, R. N. Differential Scanning Calorimetric Study of the Effect of Cholesterol 
on the Thermotropic Phase Behavior of a Homologous Series of Linear Saturated Phosphatidylcholines. Biochemistry 1993, 32, 516522.

(44) Lewis, R. N. A. H.; Mannock, D. A.; McElhaney, R. N. Differential Scanning Calorimetry in the Study of Lipid Phase Transitions in Model and Biological Membranes. Practical Considerations. In Methods in Molecular Biology; Methods in Membrane Lipids; Dopico, A. M., Ed.; Humana Press, Inc.: Totowa, NJ, 2007; Vol. 400, pp 171-195.

(45) Morrow, M. R.; Davis, J. H. Calorimetric and Nuclear Magnetic Resonance Study of the Phase Behaviour of Dilauroylphosphatidylcholine/Water. Biochim. Biophys. Acta, Biomembr. 1987, 904, 61-70.

(46) Kuhn, H.; Försterling, H.-D. Principles of Physical Chemistry: Understanding Molecules, Molecular Assemblies, Supramolecular Machines; Wiley: Chichester, U.K., 2000; pp 344-346.

(47) Shepherd, J. C. W.; Büldt, G. Zwitterionic Dipoles as a Dielectric Probe for Investigating Head Group Mobility in Phospholipid Membranes. Biochim. Biophys. Acta, Biomembr. 1978, $514,83-94$

(48) Tardieu, A.; Luzzati, V.; Reman, F. C. Structure and polymorphism of the hydrocarbon chains of lipids: a study of lecithin-water phases. J. Mol. Biol. 1973, 75, 711-733.

(49) Stern, H. A.; Feller, S. E. Calculation of the Dielectric Permittivity Profile for a Nonuniform System: Application to a Lipid Bilayer Simulation. J. Chem. Phys. 2003, 118, 3401-3412.

(50) Huheey, J. E. Inorganic Chemistry, 3rd ed.; Harper Collins: New York, 1983; pp 60-62.

(51) Lide, D. R. Handbook of Chemistry and Physics, 73rd ed.; CRC

Press: Boca Raton, FL, 1992; pp 5-94.

(52) Chen, X.; Hua, W.; Huang, Z.; Allen, H. C. Interfacial Water Structure Associated with Phospholipid Membranes Studied by Phase-Sensitive Vibrational Sum Frequency Generation Spectroscopy. J. Am. Chem. Soc. 2010, 132, 11336-11342.

(53) Mondal, J. A.; Nihonyanagi, S.; Yamaguchi, S.; Tahara, T. Three Distinct Water Structures at a Zwitterionic Lipid/Water Interface Revealed by Heterodyne-Detected Vibrational Sum Frequency Generation. J. Am. Chem. Soc. 2012, 134, 7842-7850.

(54) Nihonyanagi, S.; Mondal, J. A.; Yamaguchi, S.; Tahara, T. Structure and Dynamics of Interfacial Water Studied by HeterodyneDetected Vibrational Sum-Frequency Generation. Annu. Rev. Phys. Chem. 2013, 64, 579-603.

(55) Cyran, J. D.; Backus, E. H. G.; Nagata, Y.; Bonn, M. Structure from Dynamics: Vibrational Dynamics of Interfacial Water as a Probe of Aqueous Heterogeneity. J. Phys. Chem. B 2018, 122, 3667-3679.

(56) Peterson, U.; Mannock, D. A.; Lewis, R. N. A. H.; Pohl, P.; McElhaney, R. N.; Pohl, E. E. Origin of Membrane Dipole Potential: Contribution of the Phospholipid Fatty Acid Chains. Chem. Phys. Lipids 2002, 117, 19-27.

(57) Gawrisch, K.; Ruston, D.; Zimmerberg, J.; Parsegian, V. A.; Rand, R. P.; Fuller, N. Membrane dipole potentials, hydration forces, and the ordering of water at membrane surfaces. Biophys. J. 1992, 61, $1213-1223$.

(58) Mashl, R. J.; Scott, H. L.; Subramaniam, S.; Jakobsson, E. Molecular Simulation of Dioleoylphosphatidylcholine Lipid Bilayers at Differing Levels of Hydration. Biophys. J. 2001, 81, 3005-3015.

(59) Starke-Peterkovic, T.; Clarke, R. J. Effect of headgroup on the dipole potential of phospholipid vesicles. Eur. Biophys. J. 2009, 39, 103-110. 